\title{
Altered Epitope Expression of Human Interstitial Fluid Apolipoprotein A-I Reduces its Ability to Activate Lecithin Cholesterol Acyl Transferase
}

\author{
Laurence Wong, Linda K. Curtiss, * Juren Huang, Christopher J. Mann, Blanca Maldonado, and Paul S. Roheim \\ Louisiana State University Medical Center, Division of Lipoprotein Metabolism and Pathophysiology, Department of Physiology, \\ New Orleans, Louisiana 70112-2822; and * Research Institute of Scripps Clinic, La Jolla, California 92037
}

\begin{abstract}
In human peripheral interstitial fluid, esterification of cholesterol by lecithin cholesterol acyltransferase ( LCAT) was found to occur at a rate of only $10 \%$ of that in plasma $(5.6 \pm 1.8 \mathrm{com}-$ pared with 55.6 $\pm 7.8 \mathrm{nmol} / \mathrm{ml}$ per $\mathrm{h})$. Measurement of cholesterol esterification in the presence of excess reconstituted apoA-I HDL (rA-I HDL) revealed an LCAT activity in interstitial fluid of $24 \%$ of that in plasma, indicating that the low rate of esterification could not be caused by limiting mass of LCAT enzyme. When plasma was diluted to the same concentration as in interstitial fluid, the percent cholesterol esterification rate was the same as undiluted plasma and significantly higher than that of interstitial fluid. These findings led us to postulate that poor activation of LCAT in interstitial fluid may result from a change in conformation in apoA-I. To test this hypothesis, a monoclonal antibody AI-11 that inhibits apoA-I activation of LCAT was used to measure apoA-I in interstitial fluid and plasma. Antibody AI-11 recognized interstitial fluid apoA-I poorly, whereas a polyclonal antibody recognized interstitial fluid apoA-I normally. Incubation of antibody AI-11 with high density lipoprotein or rA-I HDL inhibited apoA-I activation of LCAT. We conclude that the altered conformation of apoA-I in interstitial fluid may render it a poor activator of LCAT. ( $J$. Clin. Invest. 1992. 90:2370-2375.) Key words: lecithin cholesterol acyl transferase $\bullet$ apolipoprotein A-I $\bullet$ interstitial fluid • epitope expression
\end{abstract}

\section{Introduction}

Lecithin cholesterol acyl transferase (LCAT) ${ }^{1}$ is an enzyme that catalyses the fatty acid esterification of cholesterol from cholesterol and phosphatidyl choline (1) and is responsible for the presence of most of the cholesteryl esters in circulating plasma. As a consequence of this reaction, cholesterol is converted into the more hydrophobic cholesteryl ester. In turn, phosphatidyl choline is changed into lysophosphatidyl choline.

Address correspondence to Laurence Wong, Ph.D., Louisiana State University Medical Center, Division of Lipoprotein Metabolism and Pathophysiology, Department of Physiology, 1542 Tulane Ave., New Orleans, LA 70112-2822.

Received for publication 18 March 1992 and in revised form 27 May 1992

1. Abbreviations used in this paper: IF/P, interstitial fluid/plasma ratio; LCAT, lecithin cholesterol transferase; rA-I-HDL, reconstituted apoA-I HDL.

J. Clin. Invest.

(C) The American Society for Clinical Investigation, Inc.

$0021-9738 / 92 / 12 / 2370 / 06 \$ 2.00$

Volume 90, December 1992, 2370-2375
This causes the cholesterol to transfer from the surface of the lipoprotein into the core (2). By facilitating this esterification of cholesterol, LCAT plays a major role in the transfer of cholesterol from the peripheral tissues to the liver, where essentially all of the cholesterol is metabolized (3). This enzyme helps to reshape $\operatorname{HDL}(4,5)$, especially the conversion of discoidal HDL to spherical ones (5), and together with cholesteryl ester transfer protein, facilitates the redistribution of lipids (cholesterol ester for triacylglycerol) between different lipoprotein classes (1).

The important events of reverse cholesterol transport occur extravascularly; i.e. within the interstitial space. To understand reverse cholesterol transport in the interstitial space, we have chosen to study lipoprotein metabolism in the prenodal peripheral lymph. This is an accepted model for interstitial fluid. During the past decade, our laboratory has been systematically characterizing lipoproteins and apolipoproteins in the peripheral lymph of dogs (6-10). In general, lymph lipoproteins have more HDL relative to LDL and very low density lipoproteins (10). The lipid content and particle size of the lymph lipoproteins are also different from their plasma counterparts. To determine the cause of the differences in lipoprotein lipid content, we have measured the activities of lipoprotein lipase and hepatic triacylglycerol lipase in both the plasma and lymph (11). Our study indicated that there is virtually no hepatic triacylglycerol lipase activity and very little lipoprotein lipase activity in the periphery (11). This suggests that the differences in lipoprotein composition in lymph could not have resulted from lipase modification.

A large proportion of dog prenodal lymph $\mathrm{HDL}$, especially from animals fed a high cholesterol diet, is discoidal (7). Since the presence of these particles is associated with familial LCAT deficiency (12), it is reasonable to assume that dog lymph contains little LCAT activity. Indeed very little activity was found (9) when LCAT activity was measured using the StokkeNorum assay (13).

Our findings using dog lymph as a model are consistent with those published by others using human lymph. A review of the subject has been published recently (14). In this report, we used a suction technique (15) to isolate human interstitial fluid. We found that human interstitial fluid LCAT activity is lower than expected based on its molecular size and the sieving characteristics of the interstitial space. Furthermore, the reason for the low LCAT activity was not caused by lower LCAT concentration. Instead, we found that lymph lipoproteins are poor substrates for LCAT.

\section{Methods}

Materials. Solvents (HPLC and American Chemical Society grade reagents) were obtained either from Fisher Scientific (Springfield, NJ) or from J. T. Baker Chemical Co. (Phillipsburg, NJ). Thin layer chroma- 
tography plates were from J. T. Baker Chemical Co. Liquid scintillation fluid and $4\left[{ }^{14} \mathrm{C}\right]$ cholesterol $(50 \mathrm{mCi} / \mathrm{mmol})$ were from Amersham Corp. (Arlington Heights, IL). All other chemicals were from Sigma Chemical Co. (St. Louis, MO).

Collection of interstitial fluid. Interstitial fluid was obtained from the scapular region of normal human volunteers using a suction device (Dermovac Instrumentarium, Helsinki, Finland), as described by Kiistala (16). Mild suction (200-250 $\mathrm{mmHg}$ ) was applied to the skin for $2 \mathrm{~h}$. After this period, the accumulated fluid $(0.3-0.6 \mathrm{ml})$ was withdrawn from the resulting blisters using a syringe equipped with a 25 gauge needle and placed on ice. All fluids were immediately adjusted to $1 \mathrm{mM}$ EDTA, $0.1 \% \mathrm{Na}$ azide, and analyzed within $24 \mathrm{~h}$.

Lecithin cholesterol acyl transferase assays. The rate of cholesterol esterification was measured using the method of Stokke-Norum (13) described previously (8). These assays were carried out immediately after the collection of suction fluid. Briefly, to $20 \mathrm{vol}$ of sample was added 1 vol of a solution containing $1 \mu \mathrm{Ci} / \mathrm{ml}$ of $\left[{ }^{14} \mathrm{C}\right]$ cholesterol complexed to $2 \%$ albumin in $50 \mathrm{mM}$ phosphate buffer, $\mathrm{pH} 7.4$. This mixture was incubated at $4^{\circ} \mathrm{C}$ for $4 \mathrm{~h}$ before incubation at $37^{\circ} \mathrm{C}$ for varying time periods. At the indicated times, the reaction was stopped by the addition of chloroform/methanol (2:1 vol/vol) together with $100 \mu \mathrm{g}$ each of carrier cholesterol and cholesteryl ester. After sedimentation of the insoluble precipitate by centrifugation, the lipid-rich supernatant was dried under nitrogen, redissolved in $n$-heptane, and spotted onto a silica gel thin layer chromatography plate. The cholesterol and cholesterol esters were separated using petroleum ether/ethyl acetate/acetic acid (85:15:1.5 vol/ vol/ vol) as developing solvent. After chromatography, these spots were visualized by iodine, scraped from the plate, and counted by liquid scintillation.

LCAT activity was measured based on the method of Jonas et al. (17), using reconstituted apoA-I high density lipoproteins (rA-I HDL). Reconstituted apoA-I HDLs were prepared using cholate/egg yolk lecithin/unesterified cholesterol/apoA-I in a molar ratio of 100:100:10:1. Interstitial fluid or plasma was incubated at $37^{\circ} \mathrm{C}$ with rA-I HDL substrate ( $12 \mu \mathrm{g}$ cholesterol, $0.04 \mu \mathrm{Ci}$ ) and 2-mercaptoethanol ( $4 \mathrm{mM}$ ) in a buffer containing $10 \mathrm{mM}$ Tris, $150 \mathrm{mM} \mathrm{NaCl}, 0.01 \%$ EDTA, 0.8\% BSA, pH 7.4. At the indicated times, the reaction was terminated and cholesterol and cholesteryl esters were extracted, separated, and counted as with the rate of cholesterol esterification assay.

LCAT was purified 12,000-fold from blood bank plasma using the method of Bonelli and Jonas (18). Briefly, total lipoproteins were removed from human plasma by ultracentrifugation. The clear middle zone between the lipoprotein and other proteins was used for chromatography on a phenyl-Sepharose column. The pooled fractions with LCAT activity were dialyzed and applied to a DEAE Sephacryl column. The eluate from the ion exchange column containing LCAT activity was pooled, dialyzed, and then subjected to a hydroxylapatite column. The eluate from the hydroxylapatite column was then dialyzed and stored for use (17). Apolipoprotein A-I was purified using a single step gel filtration of delipidated $\mathrm{HDL}_{3}$ in reduced ( 5 mM DTT) 4 $\mathrm{M}$ guanidium hydrochloride, $0.1 \mathrm{M}$ Tris- $\mathrm{Cl}, \mathrm{pH} 8.6$ buffer using a Sephacryl S-300 column (19).

ELISA. The polyclonal competitive ELISA has been described in detail in another publication (19). Briefly, HDL $(1.063<d<1.21$ $\mathrm{g} / \mathrm{ml}$ ) was coated onto microtiter plates (Nunc MaxiSorp F96; Vangard International Inc., Neptune, $\mathrm{NJ}$ ) and incubated at room temperature overnight. Polyclonal antibody and either unknown sample or plasma standard were incubated together in microfuge tubes overnight. This mixture was then introduced into wells of the ELISA plate. The HDL served to capture antibody not complexed to the ligand. This HDL-bound antibody was then localized with peroxidase-linked antibody and quantified by color reaction using a peroxidase substrate. This assay was not affected by sample deamination, length of sample storage, or sample delipidation (19).

All the monoclonal antibodies used in this paper have been described and characterized previously (20-23). To compare the binding of monoclonal antibodies AI-10 and AI-11 to apoA-I, two sandwich ELISAs were established. The polyclonal anti apoA-I used in the com- petitive ELISA was used as a capture agent. This ensured a constant quantity of apoA-I was captured before detection with the different monoclonal antibodies. For these assays, the polyclonal anti-apoA-I IgG was purified by ammonium sulfate precipitation (24) followed by DEAE Sephacryl chromatography (final concentration $30 \mu \mathrm{g} / \mathrm{ml}$ in $0.01 \mathrm{M}$ sodium phosphate, $0.145 \mathrm{M} \mathrm{NaCl}, \mathrm{pH} 7.4$ buffer containing $0.1 \%$ sodium azide). The microtiter plates were coated with $100 \mu \mathrm{l}$ of anti apoA-I IgG at $37^{\circ} \mathrm{C}$ for $2 \mathrm{~h}$. The plates were then washed three times with $\mathrm{NaCl} / \mathrm{Pi} /$ Tween buffer $(0.01 \mathrm{M}$ sodium phosphate, $\mathrm{pH} 7.4$, and $0.145 \mathrm{M} \mathrm{NaCl}$ with $0.05 \%$ Tween-20), using a plate washer (Skatron Microwash II; Lier, Norway). The remaining binding sites were blocked by adding $150 \mu \mathrm{l} /$ well of $1 \%$ BSA (Cohn Fraction V, filtered through $0.45-\mu \mathrm{m}$ filter) in $\mathrm{NaCl} / \mathrm{Pi}$ buffer and incubated at $37^{\circ} \mathrm{C}$ for 1 h. The plates were washed three times with $\mathrm{NaCl} / \mathrm{Pi} / \mathrm{T}$ ween buffer. ApoA-I in plasma and interstitial fluid (diluted sequentially from 3,000 - to 30,000 -fold with $\mathrm{NaCl} / \mathrm{Pi} / \mathrm{Tween}$ buffer) was then mixed with either the monoclonal antibody AI-10 or AI-11 (ascites fluid, diluted 6,000 -fold in $\mathrm{NaCl} / \mathrm{Pi} / \mathrm{Tween}$ ) and $100 \mu \mathrm{l}$ was dispensed per well. The plates were incubated at room temperature $\left(25^{\circ} \mathrm{C}\right)$, overnight (16-20 h), and then washed three times with $\mathrm{NaCl} / \mathrm{Pi} /$ Tween buffer. $100 \mu \mathrm{l}$ of peroxidase-labeled rabbit anti-mouse antibody (ICN Biomedicals, Inc., Costa Mesa, CA, or Zymed Labs Inc., South San Francisco, CA) diluted 500 -fold with $\mathrm{NaCl} / \mathrm{Pi}$ buffer containing $1 \%$ BSA were pipetted into each well, and the plates were incubated for $1 \mathrm{~h}$ at room temperature $\left(25^{\circ} \mathrm{C}\right)$. After incubation, the plates were washed, and $100 \mu \mathrm{l}$ of color reagent was added to each well. The color reagent contained $1.8 \mathrm{mM}$ ABTS [2',2-azino-bis (3-ethylbenzthiazoline-6-sulfonic acid), $0.003 \% \mathrm{H}_{2} \mathrm{O}_{2}, 0.1 \mathrm{M}$ sodium phosphate buffer, $\mathrm{pH}$ 6.0. Optical density was measured using an ELISA plate reader (SLT EAR 400 AT; SLT Lab Instruments, Hillsborough, NC) using a measuring wavelength of $405 \mathrm{~nm}$ and reference wavelength of $492 \mathrm{~nm}$. The data was directly imported to an IBM PC-AT compatible microcomputer (Z248; Zenith) and analyzed using software (Immunofit; Beckman Instruments, Inc., Fullerton, CA). The analysis software was based on the four-parameter curve fitting proposed by Rodbard et al (25). For the sandwich assay using monoclonal antibody AI-11, the procedures are identical except $\mathrm{NaCl} / \mathrm{Pi}$ buffer was used instead of the $\mathrm{NaCl} / \mathrm{Pi}$ / Tween buffer in all instances.

Other analytical techniques. Free and total cholesterol determination determinations were done according to the method of McGowan et al (26). Albumin and apoB concentrations were determined by rocket immunoelectrophoresis (27). Total protein was determined by the method of Lowry et al (28).

\section{Results}

The composition of human interstitial fluid obtained from the scapular region by negative pressure is summarized in Table I. These data are in agreement with values reported by others using a similar device on the upper arm (15) and are consistent with our findings reported for dog peripheral lymph (10).

An interesting observation made during investigation of dog peripheral lymph was that the ability of this fluid to esterify cholesterol was very low (9). To test whether this also occurs in human subjects, we compared the rate of cholesterol esterification of plasma and interstitial fluid. As shown in Fig. $1 A$, the rate of cholesterol esterification of plasma was considerably higher than interstitial fluid. Because plasma also contained four times more free cholesterol than interstitial fluid ( 1,195 vs $303 \mathrm{nmol} / \mathrm{ml}$ ), we determined the lowered rate of cholesteryl esterification in interstitial fluid was caused by the lower concentration of substrate lipoproteins. Results showed that plasma, diluted to $1 / 5$ of its concentration, still had a higher rate of cholesterol esterification than interstitial fluid (Fig. 1 $A$ ). However, when expressed as a percent cholesterol esterified (Fig. $1 B$ ), plasma diluted to $1 / 5$ of its original gave similar 
Table I. Interstitial Fluid to Plasma (IF/P) Ratio of Cholesterol, ApoA-I, ApoB, and Albumin

\begin{tabular}{lccccr}
\hline & Total protein & Albumin & Cholesterol & ApoA-I & ApoB \\
\hline Mean \pm SD & $0.44 \pm 0.05$ & $0.47 \pm 0.05$ & $0.25 \pm 0.05$ & $0.31 \pm 0.05$ & $0.21 \pm 0.05$ \\
Range & $0.37-0.48$ & $0.39-0.53$ & $0.19-0.31$ & $0.24-0.36$ & $0.17-0.28$
\end{tabular}

Interstitial fluid was collected from the scapular region as described in Methods. Our interstitial fluid values were similar to those previously reported (15). Values are mean and SD of six individuals. Values for apolipoproteins and albumin were obtained using rocket immunoelectrophoresis (27). The mean cholesterol value was $183 \mathrm{mg} / \mathrm{dl}$ in the plasma.
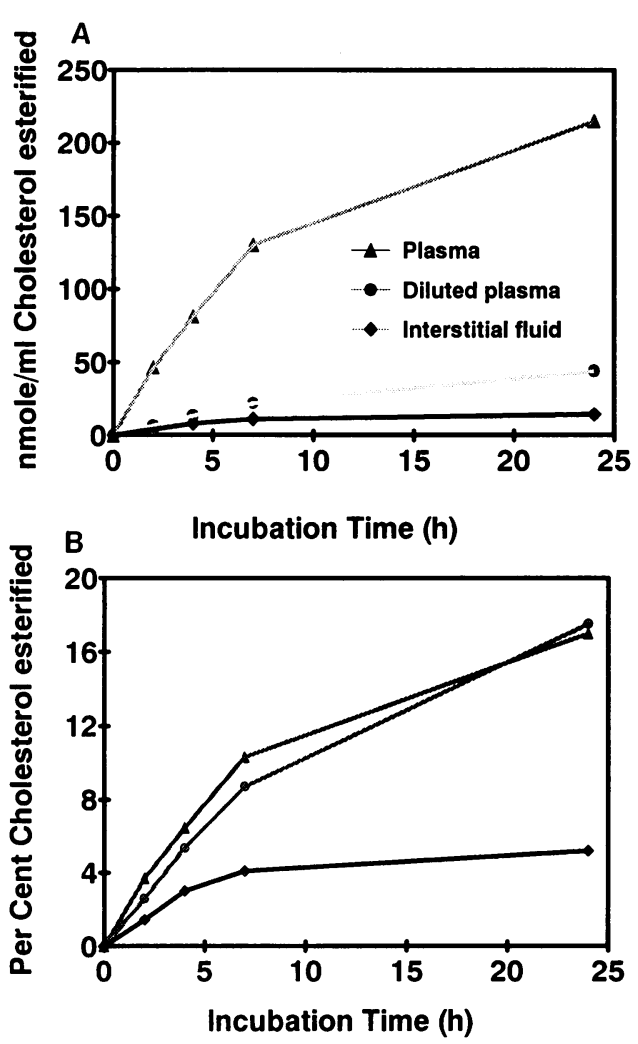

Figure $1 \mathrm{~A}$. Interstitial fluid lipoproteins are poor substrates for LCAT. Cholesterol esterification rate by LCAT in interstitial fluid, plasma, and diluted plasma were measured using the Stokke-Norum method (13), except that no dithionitrobenzoate was added to the mixture during the inclubation with radioactive cholesterol, as described in Methods. The results were expressed as nanomoles cholesterol esterified per milliliter per hour. The plasma-free cholesterol concentration was $1,195 \mathrm{nmol} / \mathrm{ml}$ while the interstitial fluid free cholesterol concentration was $303 \mathrm{nmol} / \mathrm{ml}$. Even when plasma was diluted to $1 / 5$ of its concentration, the plasma lipoproteins still had a higher rate of cholesterol esterification. This figure illustrates the absolute rate of cholesterol esterification. $(B)$ Diluted plasma has the same percent esterification rate as undiluted plasma. The data in this figure is identical to those of Fig. $1 \mathrm{~A}$, except that it is expressed as relative rates of cholesterol esterification (percent cholesterol esterified). LCAT activity was measured using the Stokke-Norum assay for interstitial fluid, plasma, and diluted plasma for an individual. This result highlights the observation, illustrated in Fig. $1 A$, that interstitial fluid lipoproteins were different from plasma lipoproteins in their ability to activate LCAT. By expressing the data this way, we also demonstrate that the differences observed between plasma and interstitial fluid are not caused by low concentration of free cholesterol in interstitial fluid. By diluting the plasma fivefold, the absolute rate of cholesterol esterification was decreased (Fig. $1 A$ ), but the relative rate of cholesterol esterification (percent esterified) was unchanged. percent of esterification as that of undiluted plasma. Thus, esterification in interstitial fluid is lower than would be expected from a simple dilution of plasma.

To test if the reduced rate of esterification in interstitial fluid could be explained by limiting concentrations of LCAT enzyme, we measured LCAT enzyme activity in the plasma and interstitial fluid using rA-I HDLs as substrate (17). Under these conditions, the amount of cholesterol esterified in interstitial fluid increased six- to sevenfold, while that in plasma increased only two- to threefold (Table II). The interstitial fluid/plasma (IF/P) ratio for LCAT increased from $10 \%$, using the endogenous substrate, to $24 \%$ using rA-I HDL, a value similar to IF/P ratio of apoA-I. These data show that the low rate of esterification in interstitial fluid is not a result of low levels of LCAT and indicate that interstitial fluid lipoproteins are poor substrates for LCAT.

Interstitial fluid lipoproteins might possibly be poor substrates for LCAT because of subtle changes in epitope expression of interstitial fluid apoA-I. Therefore, we constructed ELISAs using well defined monoclonal antibodies to probe for differences in the structure and conformation of apoA-I in interstitial fluid vs plasma.

Both monoclonal antibodies, $\mathrm{AI}-10$ and $\mathrm{AI}-11$, can be considered pan apoA-I antibodies because they bind $93-100 \%$ of plasma apoA-I (20). Monoclonal antibody AI-11 recognizes amino acids 96-111 of apoA-I (21), a region shown to be important for LCAT activation. The results of the sandwich ELISA using monoclonal antibody AI-10 as a detector antibody are shown in Fig. 2. The identical slopes for interstitial fluid and plasma demonstrated that there was no difference in the affinity of the antibody for plasma or interstitial fluid. In

Table II. Comparison of Cholesterol Esterification Activity by LCAT in Plasma and Interstitial Fluid Using Endogenous or Exogenous Substrates

\begin{tabular}{|c|c|c|c|}
\hline & \multicolumn{2}{|c|}{ Cholesterol esterified } & \multirow[b]{2}{*}{$\mathrm{IF} / \mathrm{P}$} \\
\hline & $\begin{array}{l}\text { Interstitial } \\
\text { fluid }\end{array}$ & Plasma & \\
\hline & \multicolumn{2}{|c|}{ nmol/ml per $h$} & $\%$ \\
\hline Endogenous substrate & $5.6 \pm 1.8$ & $55.6 \pm 7.8$ & 10 \\
\hline Exogenous substrate & $37.1 \pm 5.0$ & $158.1 \pm 20.4$ & 24 \\
\hline ApoA-I (mg/dl) & $33.0 \pm 14.6$ & $125.3 \pm 20.2$ & 26 \\
\hline
\end{tabular}

Determination of cholesterol esterification by LCAT in the presence of endogenous substrate (13) and excess exogenous substrate (17) in interstitial fluid and plasma. The rates of cholesterol esterification shown here were derived from the linear part of the curve shown in Fig. 1. 


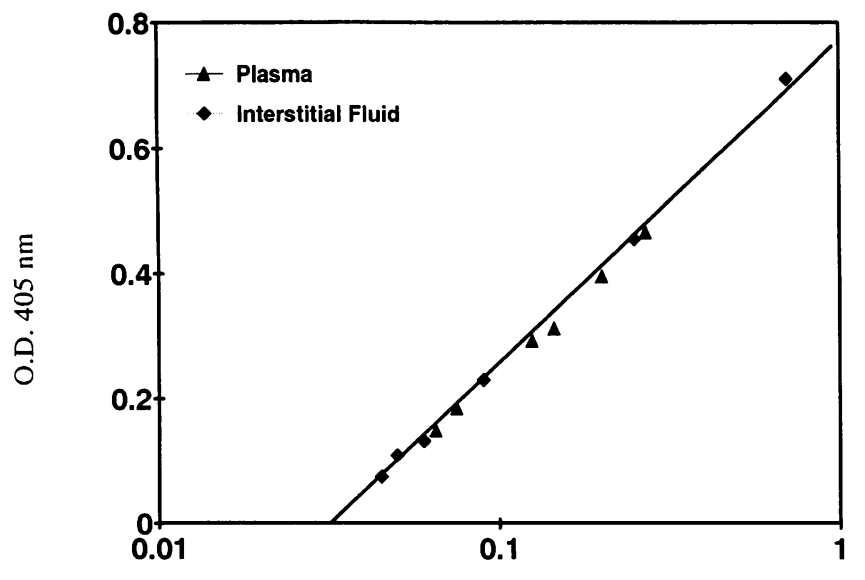

Log apo A-I $(\mu \mathrm{g} / \mathrm{ml})$

Figure 2. Monoclonal sandwich assay of AI-10 to apoA-I in interstitial fluid and plasma. A polyclonal antibody was used to capture apoA-I in plasma and interstitial fluid before probing with the monoclonal antibody. Note that the affinity of AI-10 to interstitial fluid and plasma apoA-I is similar (same slope). O.D., optical density.

contrast, when monoclonal antibody AI-11 was used as a detector, the slope for interstitial fluid apoA-I was approximately three times less than plasma (Fig. 3). Furthermore, there was a correlation between the relative affinity of AI-11 (as measured by the slope) for interstitial fluid apoA-I and the levels of cholesterol esterification in interstitial fluid (Fig. 4).

In vitro incubations, using monoclonal antibodies AI-10 or AI-11 with rA-I HDLs and purified LCAT, showed that binding of AI-11 to apoA-I was sufficient to inhibit apoA-I activation of LCAT (21). AI-11 or AI-10 was preincubated with rA-I HDLs in a 1:1 molar ratio of apoA-I to antibody $30 \mathrm{~min}$ before the addition of a purified LCAT. The monoclonal antibody AI- 11 was able to inhibit LCAT to $19 \%$ of that in control (Fig. 5 A), whereas monoclonal antibody AI-10 inhibited LCAT activation to $85 \%$ of control.

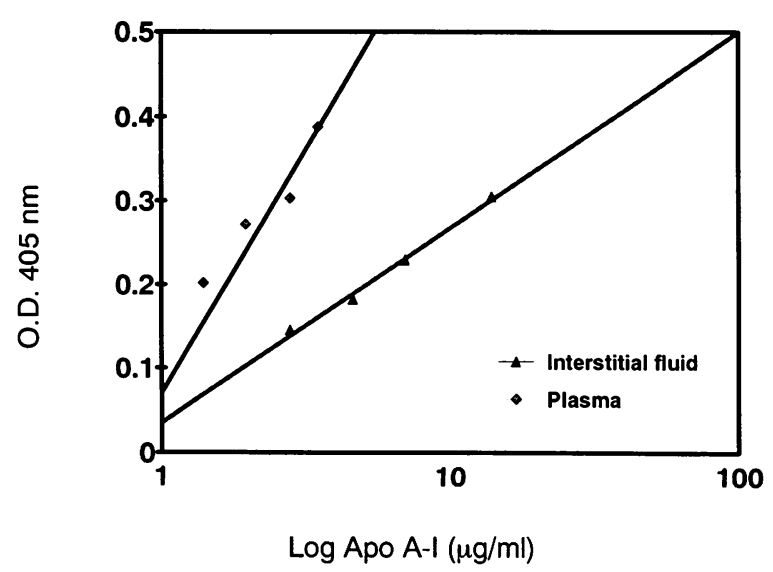

Figure 3. Monoclonal sandwich assay of AI-11 to apoA-I in interstitial fluid and plasma. A polyclonal antibody was used to capture apoA-I in plasma and interstitial fluid before probing with the monoclonal antibody AI-11. Note that the affinity of AI-11 to interstitial fluid apoA-I is less than to plasma apoA-I (different slope). Because of the difference in slope, we could not calculate the relative number of epitopes that is expressed in the interstitial fluid. O.D., optical density.

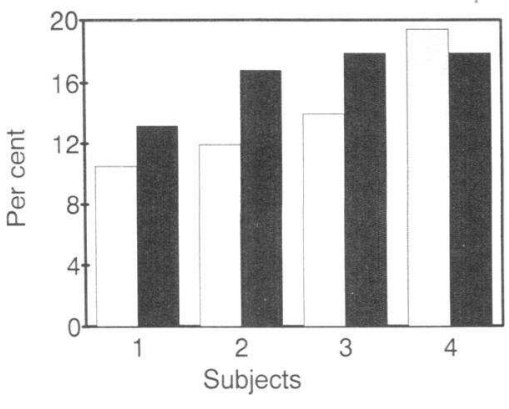

Figure 4. Relationship between relative affinity of AI- 11 to interstitial fluid apoA-I and their relative cholesterol esterification rates. The relative affinity of $\mathrm{AI}-11$ to interstitial fluid apoA-I was determined by comparing the slope of the ELISA, shown in Fig. 3, between the plasma and interstitial fluid. If the slopes were identical, then the relative affinity would be $100 \%$. This value was then paired with the relative rate of cholesterol esterification in a sample from the same subject. Note that with more affinity of AI-11 to interstitial fluid apoA-I, there is less inhibition of cholesterol esterification. This correlation is not absolute. Subjects 3 and 4 had the same rate of cholesterol esterification and yet subject 4 had a higher relative affinity, as defined by binding to AI-11. $\square$, percent relative affinity; - , percent rate of cholesterol esterification.

When plasma HDL $(1.063<d<1.21)$ was used, monoclonal antibody AI-11 was able to inhibit LCAT to $37 \%$ of control, whereas monoclonal antibody AI-10 inhibited LCAT to $84 \%$ of control (Fig. 5 B). Thus, the degree of LCAT inhibition was similar for AI-10 regardless of substrate but not for AI- 11 .

\section{Discussion}

The data presented here show that cholesterol esterification rate is low in interstitial fluid. This finding has been reported previously for human subcutaneous lymph $(29,30)$, dog cardiac and thoracic duct lymph (31), as well as dog prenodal peripheral lymph (9). The reason for the low esterification has not been elucidated. We show here that the low esterification rate is not caused by low concentrations of enzyme or substrate, but rather to a change in epitope expression of apoA-I in interstitial fluid, thus making the HDL particles poor substrates for LCAT. This particular change in epitope expression could be detected by monoclonal antibody AI-11. Recently, Banka et al. (21) showed that monoclonal antibody AI-11 could inhibit rA-I HDL activation of LCAT. Our observation confirms and extends this observation into a more physiological arena, namely interstitial fluid.

Because HDL was shown to be heterogeneous (32), we have investigated which population of interstitial HDL showed this change of epitope expression. Preliminary data suggested that $\alpha$-migrating but not pre- $\beta$-migrating HDL lack the epitope. Unfortunately, even the $\alpha$-migrating HDL were shown to be heterogeneous (32), and we currently do not know which subsets of the $\alpha$-HDL lack this epitope.

The results shown in Fig. 4 suggested that there might be a correlation between the relative affinity of AI-11 binding to interstitial HDL and its ability to activate LCAT. This is similar to the results of Banka et al. (21), who showed a correlation between binding of monoclonal antibodies and their degree of inhibition. Our results, however, suggested that at a certain level of relative affinity, any additional increase in affinity did not result in an increase in LCAT activation (see subjects 3 and 4 in Fig. 4).

The change in epitope expression is probably determined by the particle size and its lipid composition. Our previous 

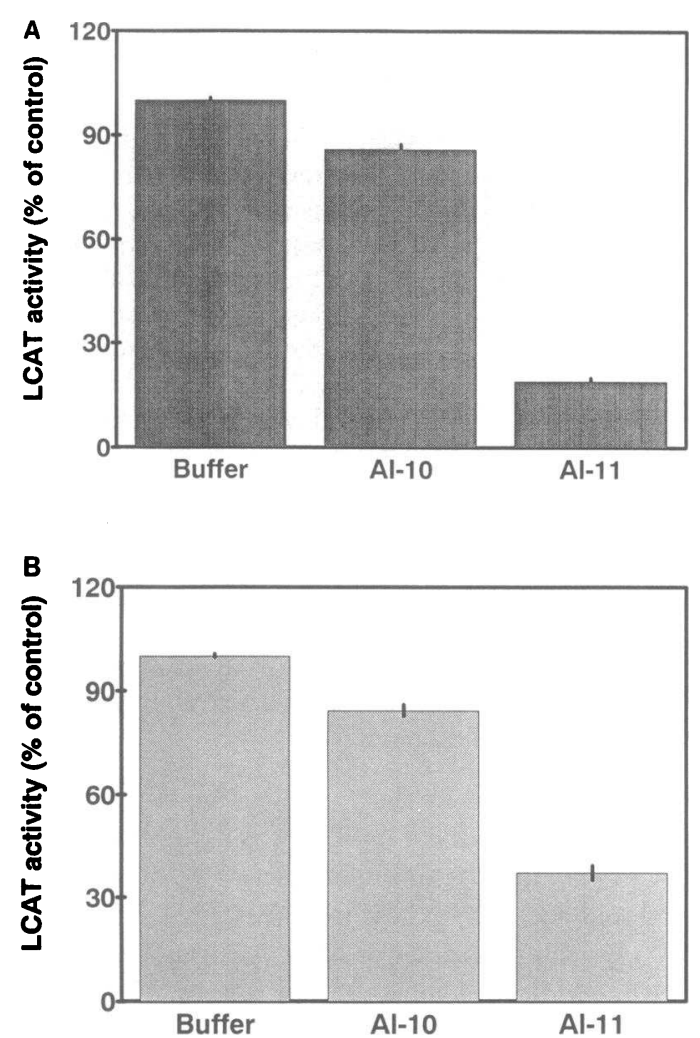

Figure $5 \mathrm{~A}$. Monoclonal antibody AI-11 inhibits LCAT activation by apoA-I containing rA-I HDLs. Reconstituted apoA-I HDLs containing radioactive cholesterol, apoA-I, and phosphatidyl choline were incubated for $30 \mathrm{~min}$ on ice in the presence or absence of monoclonal antibody AI-11 or AI-10 (1:1 molar ratio of apoA-I to antibody AI11). Purified LCAT was then added and the mixture incubated at $37^{\circ} \mathrm{C}$ for $30 \mathrm{~min}$. Esterification was measured as the rate of radioactive cholesterol ester appearance. The results are the mean and SD of duplicate determinations. ApoA-I concentration in the rA-I HDLs was $131 \mu \mathrm{g}$ and monoclonal antibody AI-10 and AI-11 were $786 \mu \mathrm{g}$. (B) Monoclonal antibody AI-11 inhibits LCAT activation by apoA-I containing HDL. HDL was incubated with a radioactive cholesterol/ albumin mixture for $4 \mathrm{~h}$ before being incubated overnight on ice in the presence or absence of monoclonal antibody AI-11 or AI-10 ( $1: 1$ molar ratio of apoA-I to antibody AI-11). Purified LCAT was then added and the mixture incubated at $37^{\circ} \mathrm{C}$ for $30 \mathrm{~min}$. Esterification was measured as the rate of radioactive cholesterol ester appearance. The results are the mean and SD of duplicate determinations. ApoA-I concentration in the HDL was $116 \mu \mathrm{g}$ and monoclonal antibody AI10 and Al-11 were $696 \mu \mathrm{g}$.

work (10) showed that interstitial fluid lipoprotein lipid composition is different from the plasma. Jonas et al. (33) have shown that by changing the molar ratio of A-I to phospholipid, and thereby changing the rA-I HDL diameter, that they could change the rA-I HDL's ability to activate LCAT. Therefore, once a population of HDL with a change in apoA-I epitope is finally identified, a detailed analysis of the lipid composition of these particles should be revealing. At present, a detailed lipid analysis of human interstitial fluid HDL subpopulations is beyond the scope of this paper. This is because of the extremely small sample of interstitial fluid that we could obtain (0.3-0.6 $\mathrm{ml}$ ), the low concentration of HDL in interstitial fluid (20$30 \%$ of plasma), and the heterogeneity of HDL subpopulations.

Through the work of Segrest et al. (34) and Pownall et al.
(35), it is generally accepted that apoA-I is not unique in its activation of LCAT. In fact, any protein, including synthetic artificial peptides, that contain an amphipathic helix could activate LCAT (35). One consequence of our investigation is the demonstration that a single epitope on apoA-I may be responsible for the activation of LCAT (see Fig. 5, $A$ and $B$, where preincubation of AI- 11 with either rA-I HDLs or HDL inhibited LCAT activation 81 and 63\%, respectively). In apoA-I, there are eight amphipathic helical 22 mers and two 11 mers. Thus, one would expect that binding of monoclonal antibody to one epitope on one helix could not inhibit LCAT activation so exclusively. One alternative explanation of our results could be that AI-11 binding to apoA-I caused a steric hindrance that obstructed other amphipathic helixes from binding to LCAT. ApoA-I has a molecular mass of $27 \mathrm{kD}$, whereas an IgG such as AI-11 has a molecular mass of $150 \mathrm{kD}$. This could not be the sole explanation, since $\mathrm{AI}-10$ did not have as dramatic an effect as AI-11. Our results are consistent with the results of Anantharamaiah et al. (36), who proposed that peptide 66-121 is the region of apoA-I that is responsible for the majority of binding to and activation of LCAT. This region, however, contained 55 amino acids consisting of two 22 mers on either side of one 11 mer. The localization of AI-11 binding to residues 96-111 would suggest that only the latter $22 \mathrm{mer}$ (amino acids 99-120 are the location of the second $22 \mathrm{mer}$ ), and a small portion of the 11 mer may be important for LCAT activation.

Our measurement of LCAT activities in interstitial fluid also provide some insight into the nature of LCAT-lipoprotein interaction when it crosses the capillary endothelium. In general, plasma proteins' concentration in interstitial fluid is inversely proportional to its Stoke's radii (37). Assuming that most proteins are globular, albumin $(67 \mathrm{kD})$ had an IF/P ratio of 0.47 (Table I), and HDL ( $200 \mathrm{kD}$ in the form of apoA-I containing lipoproteins) had an IF/P ratio of $0.24-0.36$ ( Table I). LCAT activity had an IF/P ratio of 0.23 . This corresponded to a molecular mass in the area of HDL. Therefore, our data ( Tables I and II) suggest that LCAT crosses the capillary endothelium as a HDL-LCAT complex. This data is also consistent with the existence of an apoD apoA-I LCAT complex that has been proposed (38).

Taking together the data from this paper and our previous work on low to nonexistent levels of lipases in the interstitial fluid (11), our results suggest a complex series of events in the interstitial space. Immediately after crossing the interstitial space, apoA-I may change its conformation. The change could be the result of apoA-I containing HDL crossing the interstitium. It could also be caused by the interaction of HDL with peripheral cells; for example, the composition of the phospholipid in the peripheral cell membranes may be the trigger that causes this change in apoA-I epitope. This change in conformation may facilitate removal of cholesterol from the peripheral cells to HDL. In any event, the interstitial fluid HDL are poor activators and substrates for LCAT, even though LCAT is present. Upon returning to the plasma, the interstitial fluid HDL will interact with plasma HDL. This interaction may be sufficient to change the conformation of interstitial fluid apoA-I to again make it into good substrate for LCAT. The interaction with plasma HDL could be nothing more than dilution of plasma membrane phospholipids with HDL phospholipids. Alternatively, the interstitial HDL may be recognized and metabolized differently than the plasma HDL. The action of cholesteryl ester transfer protein in this sequence of events remains to 
be determined. The exact nature of this change in epitope in apoA-I also remains to be determined.

In conclusion, the LCAT activating epitope of apoA-I, as defined by monoclonal antibody AI- 11 binding, is different in the HDL of interstitial fluid than in plasma HDL.

\section{Acknowledgments}

We wish to thank our colleagues, Dr. J. J. Thompson and Dr. C. H. Sloop for suggestions and discussion. The expert technical assistance of Shiqi Huang, Nora K. Weedy, Colleen Tierney, and Aliza Torbati is gratefully acknowledged. We also wish to thank Susan Allen for editorial assistance.

This research is supported by National Institutes of Health grants DK-35278 and HL-25596.

\section{References}

1. Fielding, C. J. 1990. Lecithin: cholesterol acyltransferase. In Advances in Cholesterol Research. M. Esfahani and J. B. Swaney, editors. Telford Press, Caldwell, NJ. 271-314.

2. Jonas, A. 1987. Lecithin: cholesterol acyltransferase. In Plasma Lipoproteins. A. M. J. Gotto, editor. Elsevier Science Publishers B.V., Amsterdam, The Netherlands. 299-333.

3. Glomset, J. A., and K. R. Norum. 1973. The metabolic role of lecithin: cholesterol acyltransferase: perspectives from pathology. Adv. Lipid Res. 2:1-65.

4. Mitchell, D. C., W. C. King, K. R. Applegate, T. Forte, J. A. Glomset, K. R. Norum, and E. Gjone. 1980. Characterization of apolipoprotein E-rich high density lipoproteins in familial lecithin: cholesterol acyltransferase deficiency. $J$. Lipid Res. 21:625-634.

5. Hamilton, R. L., M. C. Williams, C. J. Fielding, and R. J. Havel. 1976. Discoidal bilayer structure of nascent high density lipoproteins from perfused rat livers. J. Clin. Invest. 58:667-680.

6. Sloop, C. H., L. Dory, B. R. Krause, C. Castle, and P. S. Roheim. 1983. Lipoproteins and apolipoproteins in peripheral lymph of normal and cholesterolfed dogs. Atherosclerosis. 49:9-21.

7. Sloop, C. H., L. Dory, R. L. Hamilton, B. R. Krause, and P. S. Roheim. 1983. Characterization of $\operatorname{dog}$ peripheral lymph lipoproteins: the presence of a disc-shaped "nascent" high density lipoprotein. J. Lipid Res. 24:1429-1440.

8. Dory, L., L. M. Boquet, R. L. Hamilton, C. H. Sloop, and P. S. Roheim. 1985. Heterogeneity of dog interstitial fluid (peripheral lymph) high density lipoprotein: implications for a role in reverse cholesterol transport. J. Lipid Res. 26:519-527.

9. Dory, L., C. H. Sloop, L. M. Boquet, R. L. Hamilton, and P. S. Roheim. 1983. Lecithin: cholesterol acyltransferase-mediated modification of discoidal peripheral lymph high density lipoproteins. possible mechanism of formation of cholesterol-induced high density lipoproteins (HDLc) in cholesterol-fed dogs. Proc. Natl. Acad. Sci. USA. 80:3489-3493.

10. Sloop, C. H., L. Dory, and P. S. Roheim. 1987. Interstitial fluid lipoproteins. J. Lipid Res. 28:225-237.

11. Huang, J., C. H. Sloop, P. S. Roheim, and L. Wong. 1990. Lipoprotein lipase and hepatic triacylglycerol lipase activities in peripheral and skeletal muscle lymph. Arteriosclerosis J. Vasc. Biol. 10:720-726.

12. Norum, K. R., E. Gjone, and J. A. Glomset. 1989. Familial lecithin: cholesterol acyltransferase deficiency, including fish eye disease. In The Metabolic Basis of Inherited Disease. C. R. Scriver, A. L. Beaudet, W. S. Sly, and D. Valle, editors. McGraw-Hill Inc., New York 1181-1194.

13. Stokke, K. T., and K. R. Norum. 1971. Determination of lecithin: cholesterol acyltransferase in human plasma. J. Clin. Lab. Invest. 27:21-27.

14. Reichl, D., and N. E. Miller. 1989. Pathophysiology of reverse cholesterol transport-insights from inherited disorders of lipoprotein metabolism. Arterio sclerosis J. Vasc. Biol. 9:785-797.

15. Vessby, B., S. Gustafson, M. J. Chapman, K. Hellsing, and H. Lithell.
1987. Lipoprotein composition of human suction-blister interstitial fluid. $J$. Lipid Res. 28:629-641.

16. Kiistala, U. 1968. Suction blister device for separation of viable epidermis from dermis. J. Invest. Dermatol. 50:129-137.

17. Jonas, A., S. A. Swaney, and P. N. Herbert. 1984. Discoidal complexes of $A$ and $C$ apolipoproteins with lipids and their reaction with lecithin: cholesterol acyltransferase. J. Biol. Chem. 259:6369-6375.

18. Bonelli, F. S., and A. Jonas. 1989. Reaction of lecithin cholesterol acyltransferase with water-soluble substrates. J. Biol. Chem. 264:14723-14728.

19. Gaver, A., N. K. Weedy, B. R. Maldonado, S. Huang, and L. Wong. 1992. Procedure for development of a enzyme-linked immunosorbent assay. Development of an assay for human apolipoprotein A-I. Clin. Chim. Acta. 208:23-37.

20. Hogle, D. M., R. S. Smith, and L. K. Curtiss. 1988. Quantitation of plasma apo A-I using two monoclonal antibodies in an enzyme-linked immunoabsorbent assay. J. Lipid Res. 29:1221-1229.

21. Banka, C. L., D. J. Bonnet, A. S. Black, R. S. Smith, and L. K. Curtiss. 1991. Localization of an apolipoprotein-A-I epitope critical for activation of lecithin-cholesterol acyltransferase. J. Biol. Chem. 266:23886-23892.

22. Curtiss, L. K., and R. S. Smith. 1988. Localization of two epitopes of apolipoprotein A-I that are exposed on human high density lipoproteins using monoclonal antibodies and synthetic peptides. J. Biol. Chem. 263:13779-13785.

23. Curtiss, L. K., and T. S. Edgington. 1985. Immunochemical heterogeneity of human plasma high density lipoproteins. Identification with apolipoprotein A-I and A-II specific monoclonal antibodies. J. Biol. Chem. 260:2982-2993.

24. Heide, K., and H. G. Schwick. 1978. Salt fractionation of immunoglobulins. In Handbook of Experimental Immunology. D. M. Weir, editor. Blackwell Scientific Publications Ltd., Oxford, England. 7.1-7.11.

25. Rodbard, D., R. H. Lenox, H. L. Wray, and D. Ramseth. 1976. Statistical characterization of the random errors in the radioimmunoassay dose response variable. Clin. Chem. 22:350-358.

26. McGowan, M. W., J. D. Artiss, and B. Zak. 1983. Determination of free, esterified and total serum cholesterol using a sensitive color reaction. Microchem. J. 28:294-299.

27. Laurell, C.-B. 1972. Electroimmuno assay. Scand. J. Clin. Lab. Invest. 29:21-37.

28. Lowry, O. H., N. J. Rosebrough, A. L. Farr, and R. J. Randall. 1951. Protein measurement with the folin phenol reagent. J. Biol. Chem. 193:265-275.

29. Glomset, J. A. 1968. The plasma lecithin: cholesterol acyltransferase reaction. J. Lipid Res. 9:155-167.

30. Reichl, D., L. A. Simons, N. B. Myant, J. J. Pflug, and G. L. Mills. 1973. The lipids and lipoproteins of human peripheral lymph with observations on the transport of cholesterol from plasma and tissues into lymph. Clin. Sci. Mol. Med. 45:313-329.

31. Stokke, K. T., N. B. Fjeld, T. H. Kluge, and S. Skrede. 1974. Lipid composition and cholesterol esterification in lymph. Scand. J. Clin. Lab. Invest. 33:199206.

32. Castro, G. R., and C. J. Fielding. 1988. Early incorporation of cell derived cholesterol into pre- $\beta$-migrating HDL. Biochemistry. 27:25-29.

33. Jonas, A., J. H. Wald, K. L. H. Toohill, E. S. Krul, and K. E. Kezdy. 1990 Apolipoprotein-A-I structure and lipid properties in homogeneous, reconstituted spherical and discoidal high density lipoproteins. J. Biol. Chem. 265:2212322129.

34. Chung, B. H., G. M. Anantharamaiah, C. G. Brouillette, T. Nishida, and J. P. Segrest. 1985. Studies of synthetic peptide analogues of the amphipathic helix: correlation of structure with function. J. Biol. Chem. 260:10256-10262.

35. Pownall, H. J., A. Hu, A. M. J. Gotto, J. J. Albers, and J. T. Sparrow. 1980. Activation of lecithin: cholesterol acyltransferase by synthetic model lipid-association peptides. Proc. Natl. Acad. Sci. USA. 77:3154-3158.

36. Anantharamaiah, G. M., Y. V. Venkatachalapathi, C. G. Brouillette, and J. P. Segrest. 1990. Use of synthetic peptide analogues to localize lecithin: cholesterol acyltransferase activating domain in apolipoprotein A-I. Arteriosclerosis J. Vasc. Biol. 10:95-105.

37. Joyner, W. L., R. D. Carter, G. S. Raizes, and E. M. Renkin. 1974. Influence of histamine and some other substances on blood-lymph transport of plasma protein and dextran in the dog paw. Microvasc. Res. 7:19-30.

38. Fielding, C. J., and P. E. Fielding. 1980. A cholesterol ester transfer complex in human plasma. Proc. Natl. Acad. Sci. USA. 77:3327-3330. 